\title{
Determination of Depth-Dependent Intradermal Immunogenicity of Adjuvanted Inactivated Polio Vaccine Delivered by Microinjections via Hollow Microneedles
}

\author{
Pim Schipper ' Koen van der Maaden ' • Stefan Romeijn ' • Cees Oomens ${ }^{2}$ • Gideon Kersten ${ }^{\text {1,3 }}$. \\ Wim Jiskoot ' $\cdot$ Joke Bouwstra'
}

Received: 1 March 2016 / Accepted: 27 April 2016 / Published online: 17 June 2016

(C) Springer Science+Business Media New York 2016

\begin{abstract}
Purpose The aim of this study was to investigate the depthdependent intradermal immunogenicity of inactivated polio vaccine (IPV) delivered by depth-controlled microinjections via hollow microneedles (HMN) and to investigate antibody response enhancing effects of IPV immunization adjuvanted with $\mathrm{CpG}$ oligodeoxynucleotide 1826 (CpG) or cholera toxin (CT).
\end{abstract}

Methods A novel applicator for HMN was designed to permit depth- and volume-controlled microinjections. The applicator was used to immunize rats intradermally with monovalent IPV serotype 1 (IPV1) at injection depths ranging from 50 to $550 \mu \mathrm{m}$, or at $400 \mu \mathrm{m}$ for $\mathrm{CpG}$ and CT adjuvanted immunization, which were compared to intramuscular immunization.

Results The applicator allowed accurate microinjections into rat skin at predetermined injection depths (50-900 $\mu \mathrm{m})$, -volumes $(1-100 \mu \mathrm{L}$ ) and -rates (up to $60 \mu \mathrm{L} / \mathrm{min}$ ) with minimal volume loss $( \pm 1-2 \%)$. HMN-mediated intradermal immunization resulted in similar IgG and virus-neutralizing antibody titers as conventional intramuscular immunization. No differences in IgG titers were observed as function of injection depth, however IgG titers were significantly increased in the CpG and CT adjuvanted groups (7-fold).

Joke Bouwstra

bouwstra@lacdr.leidenuniv.nl

Division of Drug Delivery Technology, Cluster BioTherapeutics, Leiden Academic Centre for Drug Research, Leiden University, P.O. Box 9502, 2300 RA Leiden, The Netherlands

2 Soft Tissue Biomechanics and Engineering, Department of Biomedical Engineering, Eindhoven University of Technology, Eindhoven, The Netherlands

3 Intravacc (Institute for Translational Vaccinology), Bilthoven, The Netherlands
Conclusion Intradermal immunogenicity of IPV1 was not affected by injection depth. CpG and CT were potent adjuvants for both intradermal and intramuscular immunization, allowing effective vaccination upon a minimally-invasive single intradermal microinjection by HMN.

KEY WORDS dose sparing · hollow microneedles . intradermal immunization · inactivated polio vaccine $\cdot$ poliomyelitis

\section{ABBREVIATIONS}

$\begin{array}{ll}\text { CpG } & \text { CpG oligodeoxynucleotide } 1826 \\ \text { CT } & \text { Cholera toxin } \\ \text { DC } & \text { Dendritic cell } \\ \text { DDC } & \text { Dermal dendritic cell } \\ \text { H\&E } & \text { Hematoxylin and eosin } \\ \text { HMN } & \text { Hollow microneedle } \\ \text { IPV } & \text { Inactivated polio vaccine } \\ \text { IPVI } & \text { Monovalent inactivated polio vaccine serotype } 1 \\ \text { LC } & \text { Langerhans cell } \\ \text { OPV } & \text { Oral polio vaccine } \\ \text { PBS } & \text { Phosphate buffered saline } \\ \text { TMB } & 3,3^{\prime} 5,5^{\prime} \text {-tetramethylbenzidine } \\ \text { VN } & \text { Virus neutralizing }\end{array}$

\section{INTRODUCTION}

Poliomyelitis can be prevented through vaccination by either oral polio vaccine (OPV) or inactivated polio vaccine (IPV). Although OPV is an inexpensive and easy to administer vaccine, it may cause outbreaks of vaccine-derived polioviruses (1). Therefore, the World Health Organization aims to eliminate the use of OPV and substitute it by IPV in its goal towards worldwide polio eradication (2). However, IPV vaccination is more costly because of higher production costs and a higher dose requirement in comparison to OPV. Therefore, 
several strategies for dose sparing to reduce the cost of IPV vaccination have been proposed, including intradermal immunization and IPV adjuvantation (3).

Although intradermal IPV immunization at reduced doses in human resulted in seroconversion (4), intradermal injection by the Mantoux technique is difficult to perform. Therefore, there is an urgent need for novel intradermal injection methods. Intradermal IPV immunization via jet injectors resulted in seroconversion at reduced doses $(5,6)$. Another novel strategy for intradermal injection is microneedles, which are micron-sized needles (7). Microneedles were investigated on their ability to induce protective immune responses upon intradermal IPV delivery on rats and rhesus macaques (8-12). Hollow microneedle (HMN) mediated intradermal IPV immunization at reduced doses was also investigated in humans, which resulted in similar seroconversion rates in comparison to a full intramuscular dose (13).

Although intradermal immunization is a promising immunization strategy, it is unknown whether the immunogenicity of intradermally administered IPV is affected as a function of injection depth. This is of interest, as several classes of dendritic cells (DCs) reside in either the epidermis or the dermis. Langerhans cells (LCs) reside in the epidermis (topmost skin layer) and dermal dendritic cells (DDCs) reside in the dermis (lower layer) (14). Both LCs and DDGs have distinct immune functions and may therefore have a different role in immunization (14). Therefore, targeting different skin depths may affect the efficiency of intradermal IPV immunization. Hence, the objective of the present study is to investigate intradermal IPV immunization efficiency as function of skin injection depth by using a HMN system. To this end, precise and reproducible injection of IPV into the skin at a predefined depth is a requirement. This requirement can be fulfilled by using our previously developed in-house applicator (8). However, this applicator only allowed a maximum flow rate of $2 \mu \mathrm{L} / \mathrm{min}$ without leakage in the microinjection system. Therefore, the system was thoroughly redesigned to allow increased pressures, resulting in increased ranges of injection rates, -volumes and -depths without leakages.

Besides establishing the potential effect of intradermal injection depth on the immune response, the use of adjuvants can improve IPV immunization efficiency and therefore may result in IPV dose sparing and thus cost reduction $(3,15)$. Although colloidal aluminum hydroxide or aluminum phosphate salts have been used with IPV for intramuscular immunization (16-20), they are not for intradermal use as they cause severe side effects. Therefore, in this study we examined the immune potentiating effects of $\mathrm{CpG}$ oligodeoxynucleotide 1826 (CpG) and cholera toxin (CT) as potentially suitable adjuvants for intradermal IPV immunization.

In this study we demonstrate a HMN applicator that allows for injection depth, -rate and -volume controllable minimallyinvasive intradermal microinjections. This applicator allowed to investigate the dependence of intradermal immunogenicity to the injection depth of IPV1. To our knowledge this is the first systematic study where the influence of injection depth on antigen-specific immune responses is investigated. Additionally, the potential of adjuvants CpG and CT to enhance intradermal IPV immunization for dose sparing was investigated.

\section{MATERIALS AND METHODS}

\section{Materials}

Polyimide coated fused silica capillary $(375 \mu \mathrm{m}$ outer diameter, 100 and $20 \mu \mathrm{m}$ inner diameter) was obtained from Polymicro, Phoenix, USA. Silicone oil AK 350 was purchased from Boom Chemicals, Meppel, the Netherlands. CapTite ${ }^{\mathrm{TM}}$ connections were obtained from Labsmith, USA. Parafilm was purchased from Bemis, Monceau-sur-Sambre, Belgium. Tissue-Tek O.C.T. compound was ordered at Sakura Finetek, Alphen aan den Rijn, the Netherlands. Phosphate buffered saline, $\mathrm{pH}$ 7.4 (PBS pH 7.4: $163.9 \mathrm{mM} \mathrm{Na}^{+}$, $140.3 \mathrm{mM} \mathrm{Cl}^{-}, 8.7 \mathrm{mM} \mathrm{HPO}_{4}{ }^{2-}$ and $1.8 \mathrm{mM} \mathrm{H}_{2} \mathrm{PO}_{4}{ }^{-}, \mathrm{pH}$ 7.4) was purchased from B. Braun Melsungen, Melsungen, Germany. IsoFlo® (isoflurane 100\% w/w) was obtained from Abbott Laboratories, Maidenhead, UK. CpG was purchased from Invivogen, Toulouse, France. Hydrofluoric acid $(49 \%$ $\mathrm{w} / \mathrm{w})$, concentrated sulfuric acid (95-98\%), fluorescein, trypan blue, CT (holotoxin) and 3, 3'5, 5' tetramethylbenzidine (TMB) were obtained from SigmaAldrich, Zwijndrecht, the Netherlands. Polystyrene 96 well microtiter plates and $2.5 \mathrm{~mL}$ Vacuette ${ }^{\circledR} \mathrm{Z}$ serum separator clot activator premium tubes were ordered at Greiner BioOne, Alphen aan den Rijn, the Netherlands. Tween 80 and $30 \% \mathrm{w} / \mathrm{w}$ hydrogen peroxide were obtained from Merck, Amsterdam, the Netherlands. PBS pH $7.2\left(160.6 \mathrm{mM} \mathrm{Na}^{+}\right.$, $155.2 \mathrm{mM} \mathrm{Cl}^{-}, 2.7 \mathrm{mM} \mathrm{HPO}_{4}{ }^{2-}, 1.5 \mathrm{mM} \mathrm{H}_{2} \mathrm{PO}_{4}{ }^{-}$and $1.5 \mathrm{mM} \mathrm{K}^{+}$, pH 7.2) was purchased from Gibco (Life Technologies), Bleiswijk, the Netherlands. Protifar was obtained from Nutricia, Zoetermeer, the Netherlands. Bovine anti-poliovirus type 1 serum, monovalent IPV vaccine serotype 1 (IPV1), 1.1 M sodium acetate and $2 \mathrm{M}$ sulfuric acid were kindly provided by Intravacc, Bilthoven, the Netherlands. Horseradish peroxidase-conjugated goat-antirat IgG was obtained from Southern Biotech, Birmingham, AL, USA. Female Wistar Han IGS rats (Crl:WI(Han), strain code 273) of 175-225 g were ordered from Charles River Laboratories, Saint-Germain-sur-l'Arbresle, France.

\section{Fabrication of HMN}

HMN were produced by an in-house process as described previously (8). In short, the inner lumen of $20 \mu \mathrm{m}$ inner 
diameter polyimide coated fused silica capillaries were filled overnight with silicone oil AK 350 by use of a vacuum oven at $100^{\circ} \mathrm{C}$. These silicone oil-filled capillaries were subsequently wet etched into HMN by immersing the ends in a container with hydrofluoric acid $(49 \% \mathrm{w} / \mathrm{w})$ for $4 \mathrm{~h}$. To expose the microneedle tips, the polyimide coating at the etched ends of the capillaries was removed by immersing them in concentrated sulfuric acid $(95-98 \%)$ at $250^{\circ} \mathrm{C}$ for $5 \mathrm{~min}$.

\section{HMN Applicator}

The HMN applicator that was previously developed in-house (8) was thoroughly redesigned to enable better depthcontrolled intradermal microinjections. This HMN applicator is depicted in Fig. 1a and b. Optimization was dedicated to improve accuracy, precision and reproducibility of microinjections, to allow for increased injection rates to decrease the injection time, to allow for higher injection volumes and to achieve skin depth-controlled injections. To achieve these improvements, high-pressure resistance in the fluidics system was required. Therefore, flexible materials were replaced by nonflexible rigid materials and all previous connectors in the fluidics system were replaced by high-pressure resistant CapTite ${ }^{\mathrm{TM}}$ connectors. For the fluidics system, an $100 \mu \mathrm{L}$ Hamilton gas-tight Luer-Lock syringe (model 1710 TLL, Hamilton Robotics, Bonaduz, Switzerland) with a barrel inner diameter of $1.46 \mathrm{~mm}$ was used in conjunction with a syringe pump (NE-300, Prosense, Oosterhout, the Netherlands) and $100 \mu \mathrm{m}$ inner diameter polyimide coated fused silica capillaries.

\section{HMN Applicator Performance Validation with Ex-Vivo Rat Skin}

Microinjections into ex-vivo rat skin were performed to determine the range of injection rates, -volumes and -depths that can be used for leakage-free microinjections. Ex-vivo shaved rat skin was isolated from sacrificed female Wistar Han rats. The skin was stretched on Styrofoam covered with parafilm. Subsequently, microinjections of a solution of $10 \mu \mathrm{g} / \mathrm{mL}$ fluorescein in PBS pH 7.4 were performed at various injection rates, -volumes and -depths. To determine the accuracy and repeatability of the microinjections, volume loss that occurred at i) connections of the fluidics system, ii) on the skin surface at the injection site or iii) due to retained volume on the microneedle after its withdrawal from the injection site was measured by pipetting the lost volume with a $0.2-2 \mu \mathrm{L}$ pipette. A pipetted volume below $0.2 \mu \mathrm{L}$ was measured as a volume loss of $0.2 \mu \mathrm{L}$. The percentage volume loss was calculated as the percentage of volume loss (as measured by pipetting) from the digitally-displayed dispensed volume (as indicated by the syringe pump).
In the first series of experiments, the injection rate performance was investigated by varying injection rates from 1 to $60 \mu \mathrm{L} / \mathrm{min}$, while both the injection volume and -depth were kept constant at $10 \mu \mathrm{L}$ and at $500 \mu \mathrm{m}$, respectively. The maximum injection rate of the syringe pump for the $1.46 \mathrm{~mm}$ inner diameter $100 \mu \mathrm{L}$ Hamilton syringe was $62 \mu \mathrm{L} / \mathrm{min}$. In the second series of experiments, the injection volume was investigated by varying injection volumes from 1 to $100 \mu \mathrm{L}$, while both the injection rate and -depth were kept constant at $20 \mu \mathrm{L} / \mathrm{min}$ and at $500 \mu \mathrm{m}$, respectively. Finally, injection depths ranging from 50 to $900 \mu \mathrm{m}$ were investigated by performing $10 \mu \mathrm{L}$ microinjections at an injection rate of $20 \mu \mathrm{L} / \mathrm{min}$.

\section{Visualization of Depth-Controlled Microinjections in Ex-Vivo Rat Skin}

In order to visualize microinjections at different preselected depths in ex-vivo rat skin, trypan blue solution $(0.4 \% \mathrm{w} / \mathrm{v}$ in PBS pH 7.4) was used. Ex-vivo rat skin was isolated and prepared as described above. Next, trypan blue solution was injected at 250, 400 and $550 \mu \mathrm{m}$ depths with an injection volume of $10 \mu \mathrm{L}$ and at an injection rate of $20 \mu \mathrm{L} / \mathrm{min}$. The microinjections in ex-vivo rat skin were photographed on outer and inner skin sides by utilizing a stereo microscope at $2.5 \times$ magnification (Zeiss Stemi 2000-c, paired with a Zeiss AxioCam ICc5 camera).

Furthermore, ex-vivo rat skin injected with $0.5 \mu \mathrm{L}$ trypan blue solution at the same injection depths and at an injection rate of $1 \mu \mathrm{L} / \mathrm{min}$ was embedded in Tissue-Tek O.C.T. compound and subsequently snap-frozen in liquid nitrogen, from which $10 \mu \mathrm{m}$ thick cryosections were made on a Leica CM3050s cryostat. Subsequently, unstained and hematoxylin and eosin (H\&E) stained images were made to visualize the injection depth of the microinjections. Images were made with a Zeiss 10× plan-Apochromat objective mounted onto a Zeiss Axio Imager D2 microscope coupled with a MRc5 camera.

\section{Investigation of Depth-Dependent Intradermal Immunogenicity}

Two immunization studies were performed under the guidelines and regulations enforced by the animal ethic committee of the Netherlands, and were approved by the "Dierexperimentencommissie Universiteit Leiden (UDEC)" under number 12084. Female Wistar Han rats with a weight of 175-225 g on arrival were accommodated under standardized conditions in the animal facilities of the Leiden Academic Centre for Drug Research, Leiden University. The animals were housed in groups of 5 and were assigned to different immunization groups (10 rats per immunization group). Prior to blood withdrawal or immunization, the rats were anaesthetized with isoflurane. Anaesthetized animals that 
were assigned to the intradermal injection groups were shaved minimally (an area of $4 \mathrm{~cm}^{2}$ on the left flank) prior to the intradermal injection.

To investigate the depth-dependent intradermal immunogenicity of IPV1, the rats were immunized intradermally via HMN mediated microinjections of $10 \mu \mathrm{L}$ containing $5 \mathrm{DU}$ of IPV1 at an injection rate of $20 \mu \mathrm{L} / \mathrm{min}$ and at injection depths of 250, 400, $550 \mu \mathrm{m}$ for animal study 1 , and 50, 150, $250 \mu \mathrm{m}$ for animal study 2. As a control in both animal studies, $5 \mathrm{DU}$ of IPV1 in $200 \mu \mathrm{L}$ PBS pH 7.4 was administered intramuscularly divided over each hind leg $(100 \mu \mathrm{L}$ per hind leg). Furthermore, both animal studies contained a mock treated group via the intramuscular route $(100 \mu \mathrm{L}$ PBS pH 7.4 per hind leg). In the first animal study the immunogenicity enhancing effects of adjuvants were investigated: two groups were immunized intradermally via $\mathrm{HMN}$ mediated microinjections of $10 \mu \mathrm{L}$ (injection rate $20 \mu \mathrm{L} / \mathrm{min}$ and injection depth $400 \mu \mathrm{m}$ ) and two groups were immunized intramuscularly $(100 \mu \mathrm{L}$ per hind leg) with 5 DU IPV1 adjuvanted with either CpG or CT. These previously described immunization procedures were performed at day 1 (prime immunization) and were repeated at day 21 (booster immunization). Collection of blood samples were performed at day 1, day 21 (prime) and day 42 (boost). Blood samples were collected in $2.5 \mathrm{~mL}$ Vacuette ${ }^{\circledR}$ tubes and stored on ice before centrifugation at $2000 \mathrm{~g}$ for $10 \mathrm{~min}$ to isolate serum.

\section{Serum IgG Titers}

To measure serum IgG titers, a capture ELISA was performed. Bovine anti-poliovirus type 1 serum in PBS pH 7.2 was used to coat polystyrene 96 well microtiter plates overnight at $4^{\circ} \mathrm{C}$. Subsequently, washing was performed with 0.05\% Tween 80 in tap water. Afterwards, assay buffer consisting of PBS pH 7.2, 0.5\% (w/v) Protifar and 0.05\% (v/v) Tween 80 was used to add 4.5 DU IPV1/well $(100 \mu \mathrm{L} /$ well $)$. Plates were incubated at $37^{\circ} \mathrm{C}$ for $2 \mathrm{~h}$ before a wash step was performed. Afterwards, threefold serial dilutions of serum samples in assay buffer were added at $100 \mu \mathrm{L} /$ well and subsequently incubated at $37^{\circ} \mathrm{C}$ for $2 \mathrm{~h}$. Plates were washed before horseradish peroxidase-conjugated goat-antirat $\mathrm{IgG}$ was added to the wells (4000-fold dilution, $100 \mu \mathrm{L} /$ well) and subsequently incubated at $37^{\circ} \mathrm{C}$ for $1 \mathrm{~h}$. Subsequently, plates were thoroughly washed before adding TMB substrate solution $(100 \mu \mathrm{L} /$ well $)$ which consisted of 1.1 M sodium acetate, $100 \mathrm{mg} / \mathrm{mL}$ TMB and $0.006 \%$ (v/v) hydrogen peroxide. $2 \mathrm{M}$ sulfuric acid was used after $10 \mathrm{~min}$ to stop the reaction $(100 \mu \mathrm{L} /$ well $)$. Finally, sample absorbance was measured at $450 \mathrm{~nm}$ by a Biotek ELx808 plate reader (Winooski, VT, USA).

The Biotek Gen5 2.0 data analysis software was used to determine endpoint titers by 4-parameter analysis. The endpoint titer was defined as the reciprocal of the serum dilution producing a $450 \mathrm{~nm}$ absorbance equal to that of the mean $450 \mathrm{~nm}$ absorbance with addition of three times the standard deviation of eight samples of IPV 1-specific-antibody negative serum samples.

\section{Virus-Neutralizing Antibodies}

Determination of the antibody titers able to neutralize wildtype poliovirus serotype 1 was outsourced to Bilthoven Biologicals and performed as previously described $(21,22)$. In short, average virus-neutralizing (VN) antibody titers were measured after pooling of the serum samples of all rats per immunization group. Subsequently, two-fold serial dilutions of the pooled sera were made $\left(2^{11}-2^{22}\right)$ after inactivation of sera at $56^{\circ} \mathrm{C}$ for 30 min prior to testing. 100 TCID $_{50}$ of the Mahoney wild-type strain (poliovirus type 1) was added to the resulting serum dilutions and these virus/serum mixtures were incubated for $3 \mathrm{~h}$ at $36^{\circ} \mathrm{C}$ and $5 \% \mathrm{CO}_{2}$ before incubation at $4^{\circ} \mathrm{C}$ overnight. Subsequently, to each sample $1 \times 10^{4}$ Vero cells were added and the resulting mixtures were incubated for 7 days at $36^{\circ} \mathrm{C}$ and $5 \% \mathrm{CO}_{2}$. Finally, samples were fixed with formalin, stained with crystal violet and were analyzed macroscopically. VN titers were displayed as the last serum dilution which did not exhibit cytopathogenic effects.

\section{Statistical Analysis}

Statistics were performed using GraphPad Prism (v.6.00, GraphPad Software, LaJolla, CA, USA). Kruskall-Wallis tests with Dunn's post-hoc tests were performed as IgG titers were non-normally distributed and considered significant at $p<0.05$.

\section{RESULTS}

\section{HMN Applicator Optimization and Performance}

To allow for increased injection rates and thereby short injection times, connections in the fluidics system of the HMN applicator were optimized by applying high-pressure resistant CapTite $^{\mathrm{TM}}$ connectors and high-pressure resistant polyimide fused silica capillaries, as shown in Fig. la and b. Furthermore, the dead volume in the fluidics system was kept to a minimum to maximize injection volume accuracy and minimize the loss of vaccine formulation. The hydrofluoric acid etching procedure of polyimide coated fused silica capillaries resulted in sharp HMN (Fig. 1c).

The performance of the fluidics system was examined by varying the injection rate during intradermal microinjections into ex-vivo rat skin, mimicking back pressure during actual microinjections on live animals. As shown in Fig. 2a, the volume loss was $\pm 1-2 \%$ for all investigated injection rates at an 


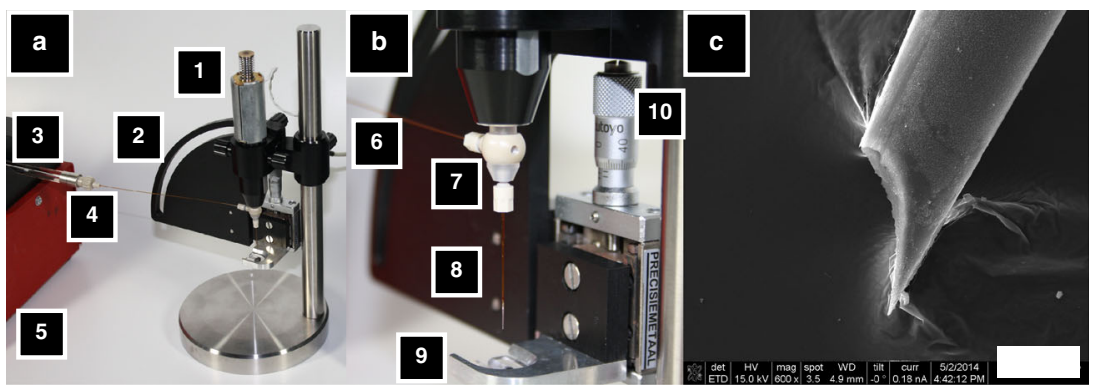

Fig. I Images of the HMN applicator (a and $\mathbf{b}$ ) and a scanning electron microscopy image of a HMN (c), bar represents $50 \mu \mathrm{m})$. The microneedle insertion speed (I to $3 \mathrm{~m} / \mathrm{s}$ ) is controlled by an electromagnet (I). Angled injections are possible via a guided rail (2). The injection depth of the microneedle into the skin is accurately controlled by a micrometer actuator ( 10 ) and a guide plate (9). Fluid flow starts with a $100 \mu \mathrm{L}$ Hamilton gas-tight Luer-Lock syringe (3) which is driven by a controllable syringe pump (5) that is connected to a $100 \mu \mathrm{m}$ inner diameter capillary (6) via a Luer-Lock-CapTite adapter (4). This capillary feeds the fluid flow via a specially designed connection piece (7) into a $\mathrm{HMN}(8)$ that enables the intradermal injections. Because the syringe pump is programmable, injection rates and -volumes can be accurately controlled.

injection volume and -depth of $10 \mu \mathrm{L}$ and $500 \mu \mathrm{m}$, respectively. Volume loss was only observed at the skin surface of the microinjection sites or on the HMN after retracting the microneedle from the skin. No additional volume loss was observed. Owing to the achieved increase in injection rate, a significant reduction in injection time was achieved.

As shown in Fig. 2b, volumes up to $100 \mu \mathrm{L}$ could be injected into ex-vivo rat skin with volume loss that was $\pm 1-$ $2 \%$, independent of injection volume, at an injection depth and -rate of $500 \mu \mathrm{m}$ and $20 \mu \mathrm{L} / \mathrm{min}$, respectively. This volume loss was observed on the skin surface at the microinjection sites.

Finally, variation in injection depth was studied by injecting $10 \mu \mathrm{L}$ in ex-vivo rat skin at different injection depths. As shown in Fig. 2c, microinjections were performed between 50 and $900 \mu \mathrm{m}$ injection depths. At all investigated injection depths, all microinjections resulted in $\pm 1-2 \%$ volume loss. Moreover, there was no increase in volume loss at any particular injection depth.

\section{Visualization of Depth-Controlled Microinjections in Ex-Vivo Rat Skin}

To obtain evidence that skin layers at different preselected depths were targeted, microinjections of a trypan blue solution were performed on ex-vivo rat skin, which was then photographed at both sides (Fig. 3a). Whereas the trypan blue spot of the shallower microinjection at a skin injection depth of $250 \mu \mathrm{m}$ was more clearly visible at the outer side of the skin, the trypan blue spot of the deeper injection at $550 \mu \mathrm{m}$ was hardly visible. Contrarily, the trypan blue spot of the deeper microinjection at a skin injection depth of $550 \mu \mathrm{m}$ was more clearly visible at the inner side of the skin than the trypan blue spot of the shallower injection $(250 \mu \mathrm{m})$. Moreover, as shown in Fig. 3b, visualization of the skin injection depth in crosssectioned skin indicated that the microinjections were indeed performed at different pre-defined skin injection depths.

\section{Injection Depth-Dependent Intradermal Immunogenicity}

To assess whether injection of IPV1 at different injection depths in the skin affects IPV1-specific antibody responses, two immunization studies in rats were conducted. Intradermal immunization was performed using the HMN applicator in a depth-controlled manner and this was compared to intramuscular immunization with a conventional 26G hypodermic needle. IPV1-specific IgG titers obtained after intradermal immunization with non-adjuvanted IPV1 at injection depths ranging between 50 and $550 \mu \mathrm{m}$ are shown in Fig. 4. Three weeks after prime immunization (Fig. 4a) and boost immunization (Fig. 4b), no significant differences in IPV1-specific IgG titers were observed at different injection depths. Moreover, IPV1-specific IgG titers obtained after intradermal immunization were similar to those obtained after intramuscular IPV1 immunization. No IPV1-specific antibody responses were observed in the mock treated group.

\section{Immunogenicity Enhancing Effects of Adjuvants CpG and CT}

To assess the potential increase in antibody responses and the applicability for use as intradermal adjuvants, CpG and CT were used as adjuvants in intradermal and intramuscular IPV immunization (Fig. 4a and b). Intradermal IPV1 immunization adjuvanted with $\mathrm{CpG}$ led to statistically significant increased IgG titers in comparison to non-adjuvanted 400 and $550 \mu \mathrm{m}$ injection depths and intramuscular IPV1 group after prime immunization. Contrarily, intramuscular IPV1 immunization adjuvanted with $\mathrm{CpG}$ did not result in any significant increase in IgG titer after prime immunization. After boost immunization however, IPV1 immunization adjuvanted with CpG via both the intradermal and intramuscular route resulted in significantly increased IgG titers in comparison to the non-adjuvanted intramuscular IPV1 group. 

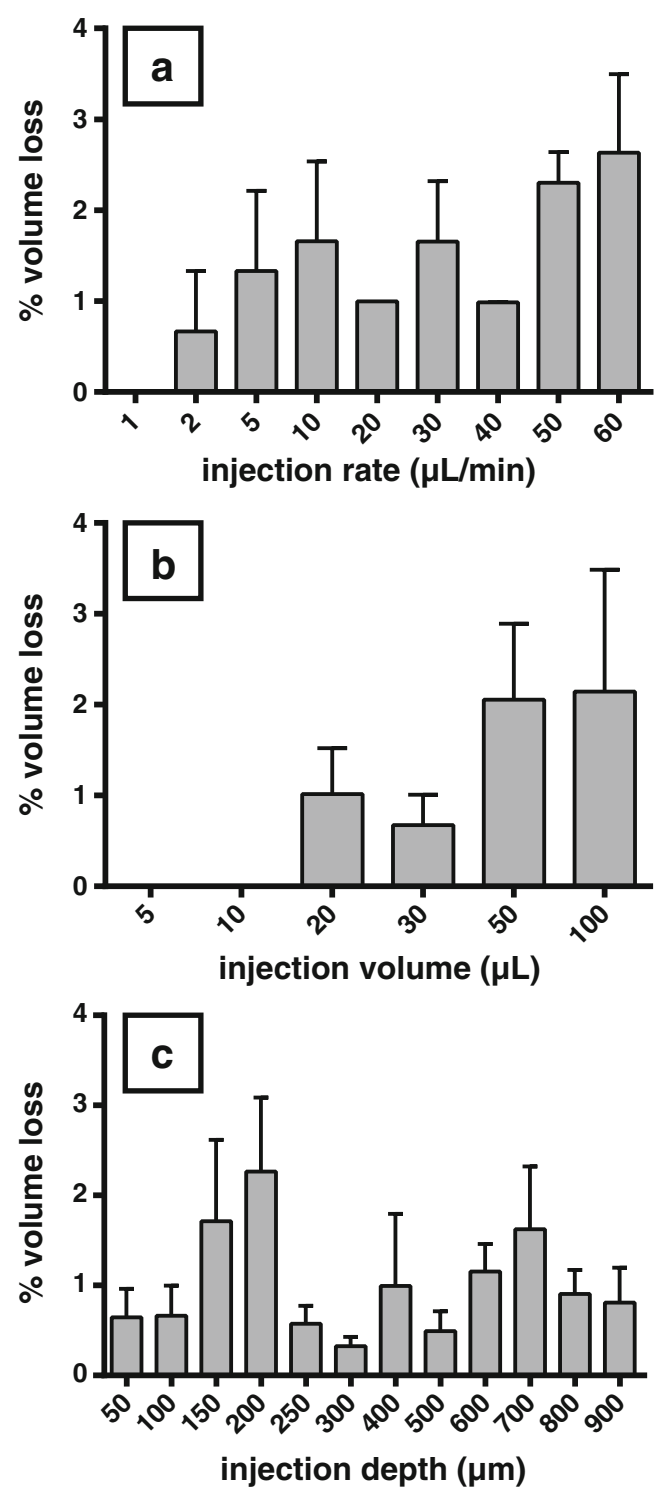

Fig. 2 Accuracy of the HMN applicator, expressed as percent volume loss, as function of (a) injection rate $(1-60 \mu \mathrm{L} / \mathrm{min}$ (pump-syringe combination maximum)) at constant injection depth $(500 \mu \mathrm{m})$ and volume (I0 $\mu \mathrm{L}) ;(\mathbf{b})$ injection volume $(5-100 \mu \mathrm{L})$ at constant injection depth $(500 \mu \mathrm{m})$ and rate (20 $\mu \mathrm{L} / \mathrm{min})$; and (c) injection depth (50-900 $\mu \mathrm{m})$ at constant injection volume $(10 \mu \mathrm{L})$ and rate $(20 \mu \mathrm{L} / \mathrm{min})$. Bars represent mean $\pm \operatorname{SEM}(n=6)$.

Intradermal IPV1 immunization adjuvanted with CT led to statistically significant increased $\mathrm{IgG}$ titers after prime immunization (non-adjuvanted $400 \mu \mathrm{m}$ injection depth) and booster immunization (non-adjuvanted intramuscular control group). Intramuscular IPV1 immunization adjuvanted with $\mathrm{CT}$ resulted in statistically significant increased IgG titers in comparison to all nonadjuvanted groups $(250,400$ and $550 \mu \mathrm{m}$ injection depths and intramuscular IPV1 group) after prime immunization. However, after boost immunization there were no significant differences for intramuscular IPV1 immunization adjuvanted with CT.
Furthermore, there were no statistically significant differences in IgG titers between $\mathrm{CpG}$ and $\mathrm{CT}$ adjuvanted groups, neither for the intradermal or the intramuscular immunization routes and neither after prime or boost immunization. However, some differences were observed between $\mathrm{CpG}$ and CT, when they were compared against non-adjuvanted groups. After prime intradermal immunization, $\mathrm{CpG}$ and $\mathrm{CT}$ adjuvanted IPV1 immunization resulted in 6.9 and 7.3 fold increased $\operatorname{IgG}$ titers on average, respectively, in comparison to the non-adjuvanted intradermal IPV1 groups. After prime intramuscular immunization, CpG and CT adjuvanted IPV1 immunization resulted in 2.7 and 12.1 fold increased IgG titers, respectively, in comparison to the non-adjuvanted intramuscular IPV1 group. Thus in prime immunization, $\mathrm{CpG}$ enhanced IgG titers more in intradermal immunization in comparison to intramuscular immunization, whereas CT enhanced IgG titers in both immunization routes. After the booster intradermal immunization, $\mathrm{CpG}$ and $\mathrm{CT}$ adjuvanted IPV1 immunization resulted in 1.9 and 2.2 fold increased IgG titers on average, respectively, in comparison to the non-adjuvanted intradermal IPV1 groups. After the booster intramuscular immunization, CpG and CT adjuvanted IPV1 immunization resulted in 5.5 and 2.4 fold increased $\operatorname{IgG}$ titers, respectively, in comparison to the non-adjuvanted intramuscular IPV1 group. Thus, the IgG titer enhancing effect was more pronounced in prime immunization than in booster immunization. Intramuscular IPV 1 immunization adjuvanted with $\mathrm{CpG}$ resulted in the strongest increase in $\mathrm{IgG}$ titers in booster immunization.

Besides increasing IgG titers, intradermal IPV1 immunization adjuvanted with $\mathrm{CpG}$ and both intradermal and intramuscular IPV1 immunization adjuvanted with CT, resulted in less low-responders in comparison to the non-adjuvanted groups after prime immunization. Moreover, these adjuvanted groups resulted in IPV1specific IgG titers after a single immunization that were comparable to the ones obtained after two nonadjuvanted immunizations.

\section{Virus-Neutralizing Antibodies}

In addition to the determination of the IPV1-specific IgG antibody responses by ELISA, protectivity of these antibodies against wildtype poliovirus was measured in a wildtype poliovirus-neutralizing antibody assay. VN antibody titers were observed in all IPV1 immunization groups (Table I). No VN antibody titer was observed in the mock treated group. Although the VN titers were measured on pooled serum samples, some slight differences in mean $\mathrm{VN}$ titer per immunization group were observed. For example, for the 
Fig. 3 Visualization of depthcontrolled microinjections of trypan blue solution by $\mathrm{HMN}$ into rat skin at $10 \mu \mathrm{L}$ and $20 \mu \mathrm{L} / \mathrm{min}(\mathbf{a})$, visualized by light microscopy at $4 \times$ magnification; bars represent $500 \mu \mathrm{m}$. Visualization of crosssections of cryofixed rat skin after depth-controlled microinjections of trypan blue solution into rat skin at $0.5 \mu \mathrm{L}$ at $10 \mu \mathrm{L} / \mathrm{min}$ were performed (b), visualized either before or after H\&E staining by bright-field microscopy at I0X magnification; bars represent $200 \mu \mathrm{m}$.

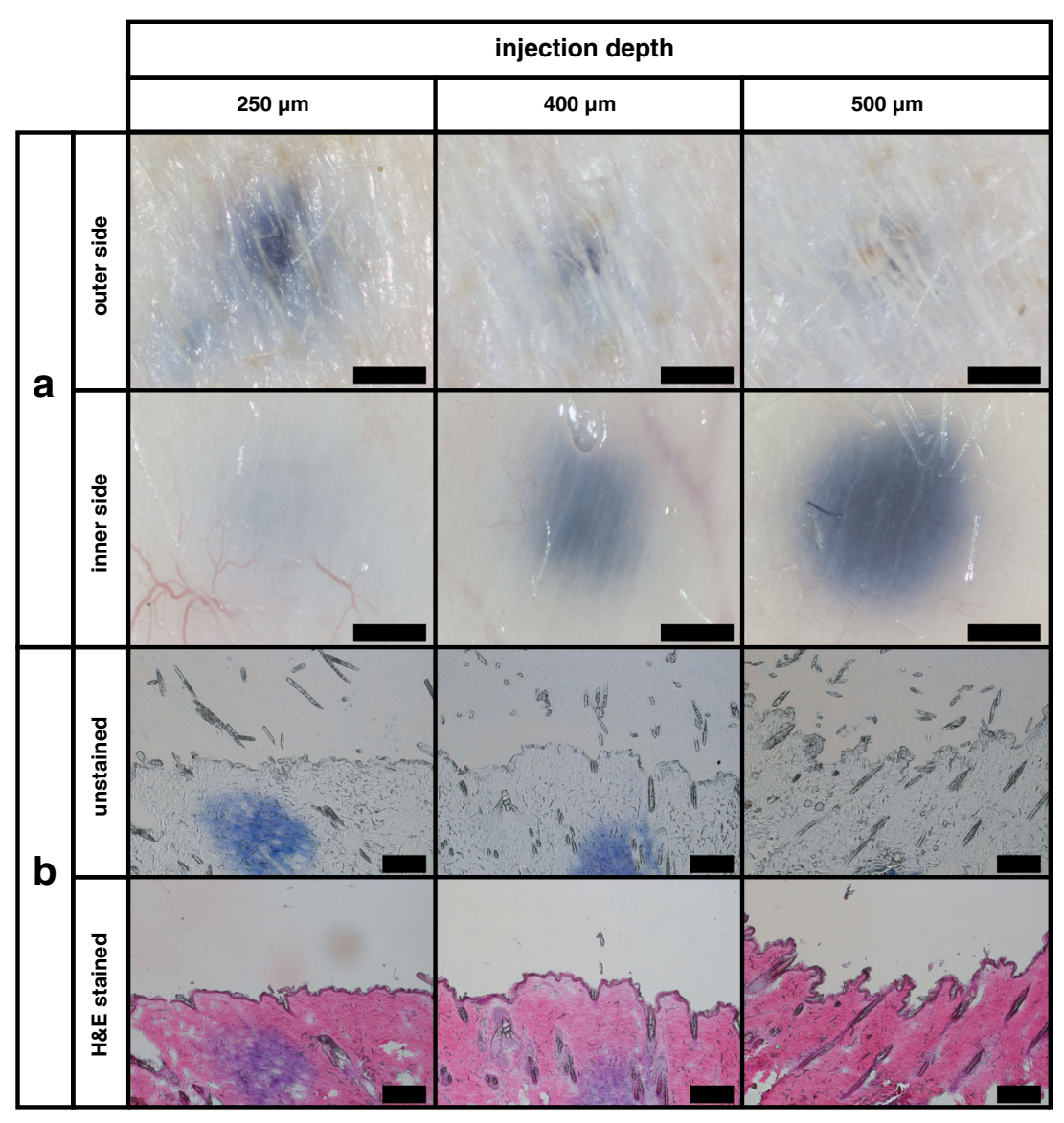

$400 \mu \mathrm{m}$ injection depth, a VN titer of 13 was observed, which was higher than those of the other injection depths and the intramuscular IPV1 immunization. Moreover, this VN titer was similar to the VN titers of intradermal and intramuscular IPV1 immunization adjuvanted with $\mathrm{CpG}$, however lower than the VN titer of intradermal IPV1 immunization adjuvanted with CT, which had the highest VN titer. VN titers of non-adjuvanted intradermal and intramuscular IPV1 immunization were similar. Intramuscular IPV1 immunization adjuvanted with $\mathrm{CT}$ resulted in a similar VN titer as non-adjuvanted intradermal and intramuscular immunization and was lower in comparison to intradermal IPV 1 immunization adjuvanted with CT. Thus, CpG enhanced VN antibody titers in both intradermal and intramuscular IPV1 immunization, whereas CT only in intradermal IPVI immunization.

Both during and immediately after intradermal immunization with HMN, a small bleb was observed on the skin. This bleb disappeared within $5 \mathrm{~min}$ after injection. No erythema was observed during and after intradermal injections with HMN, with one exception. Mild erythema was observed after intradermal injection of IPV1 with CT. This effect was only observed after the prime immunization and started $60 \mathrm{~min}$ post injection and lasted up to 7 days. Other adverse effects were not observed.

\section{DISCUSSION}

The relationship between injection depth and intradermal immunization has not been studied before, because this requires accurate, precise and reproducible depth-controlled intradermal injections with small injection volumes. For these reasons, an HMN applicator was thoroughly redesigned and improved in this study. This improved HMN applicator allowed for accurately controllable injection rates, -volumes and -depths, which allowed to investigate the depthdependent intradermal immunogenicity of IPV1, in an effort for IPV1 dose sparing.

Skin resistance during injection might significantly inhibit injection rate and accuracy (23). However, the redesigned applicator allowed for a 30-fold increased injection rate $(60 \mu \mathrm{L} / \mathrm{min})$ compared to previously achieved rates $(2 \mu \mathrm{L} /$ min) (8). Increased injection rates were needed to increase injection accuracy and shorten injection duration. Achieved injection rates were higher than those reported in literature for rat skin with a single $\mathrm{HMN}(0.17 \mu \mathrm{L} / \mathrm{min} / \mathrm{HMN}, 1 \mu \mathrm{L} / \mathrm{min} /$ HMN after needle retraction, (24)) or with HMN arrays $(0.001 \mu \mathrm{L} / \mathrm{min} / \mathrm{HMN},(25))$ and for human skin with a single HMN $(0.25-1.6 \mu \mathrm{L} / \mathrm{min} / \mathrm{HMN})$, which required hyaluronidase and needle retraction to increase rates to $18.83 \mu \mathrm{L} / \mathrm{min} /$ HMN (23). In contrast, by using the improved applicator, no 


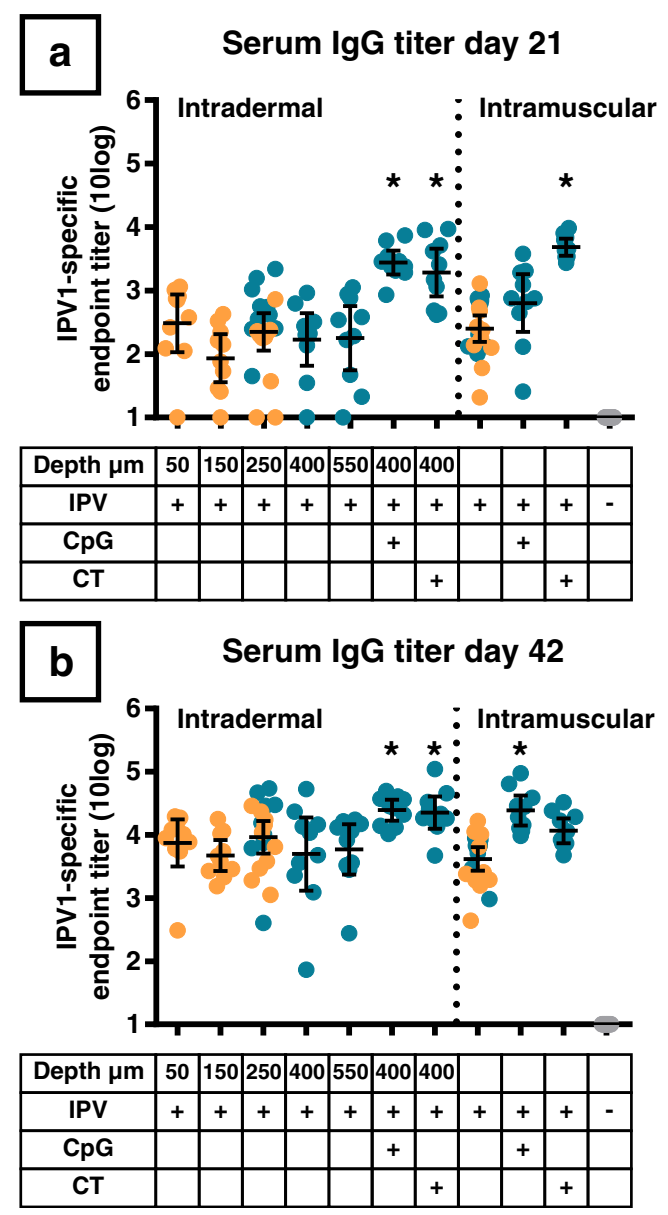

Fig. 4 IPVI-specific IgG antibody responses measured in serum obtained in study I or 2, after prime immunization (a, day 2 I) or boost immunization (b, day 42) with PBS pH 7.4, 5 DU IPVI or 5 DU IPVI adjuvanted with either $\mathrm{CpG}$ or CT. Formulations were injected intradermally via $\mathrm{HMN}$ at the indicated injection depths or intramuscularly via conventional 26G needles. Aqua or orange rounds represent animals from study I or 2, respectively. Bars represent mean $\pm 95 \%$ confidence interval $(n=10)$ and stars represent a significant increase $(p<0.05)$.

Table I Virus Neutralizing (VN) Antibody Titers Measured in Serum Obtained in Study I and 2 After Prime and Boost Immunization (Day 42) with PBS pH 7.4, 5 DU IPVI or 5 DU IPVI Adjuvanted with Either CpG or $\mathrm{CT}$. Formulations Were Injected Intradermally via $\mathrm{HMN}$ at Indicated Injection Depths or Intramuscularly via Conventional 26G Needles. Presented Data Represents VN Titers Which Were Measured on Pooled Serum Samples of All Rats Per Immunization Group

\begin{tabular}{|c|c|c|c|c|c|c|c|c|}
\hline \multirow[b]{2}{*}{ Depth $(\mu \mathrm{m})$} & \multicolumn{7}{|c|}{ Intradermal } & Intramuscular \\
\hline & 50 & 150 & 250 & 400 & 550 & 400 & 400 & \\
\hline IPVI & + & + & + & + & + & + & + & $+\quad+\quad+$ \\
\hline CpG & & & & & & + & & + \\
\hline $\mathrm{CT}$ & & & & & & & + & + \\
\hline VN titer study I & & & | | & 13 & 11 & 13 & 14 & 1213110 \\
\hline VN titer study 2 & 11 & 12 & 12 & & & & & 11 \\
\hline
\end{tabular}

additional methods were required to achieve even higher injection rates. Therefore, the utilization of non-flexible materials allowed increased pressure during injection, which allowed to easily overcome skin resistance and to achieve increased injection rates.

In addition, injection volumes achieved with this improved applicator were increased 11 -fold in comparison to those previously achieved in rat skin $(9 \mu \mathrm{L} / \mathrm{HMN})(8)$. These volumes were also much higher as those reported for single HMN in human skin $(20 \mu \mathrm{L} / \mathrm{HMN})(23)$ or HMN arrays in mice skin $(0.22 \mu \mathrm{L} / \mathrm{HMN})$ (26). Additionally, achieved volumes were comparable to HMN arrays in porcine skin $(83.33 \mu \mathrm{L} /$ HMN) (27). Furthermore, a single hollow needle design reduces the risk of volume loss (dose loss) compared to HMN arrays, as in arrays all needles need to be penetrated and unclogged to ensure an accurate injection and prevent leakage $(28,29)$.

The improved applicator allowed for an increased injection depth range $(50-900 \mu \mathrm{m})$ compared to the previously reported range $(100-400 \mu \mathrm{m})(8)$. This injection depth range was larger than the previously reported depth range in hairless rat skin (150-770 $\mu \mathrm{m},(24))$ and smaller than the previously reported depth range in human skin (180-1080 $\mu \mathrm{m},(23))$. However, these studies do not report the influence of injection depth on antigen-specific immune responses.

In contrast to our hypothesis, there was no injection depthdependent intradermal immunogenicity observed for IPV1. However, the limited thickness $(20-30 \mu \mathrm{m})$ of rat epidermis prevented injection into the epidermis where LCs reside (30). The attempt to inject IPV1 as close to the epidermis as possible did not result in a significant change in IPV 1-specific IgG titers. It is not known whether the distribution of the injected volume in the skin influences the targeting of intradermal injection depths. Furthermore, it is unclear whether local changes in innate immunity can be expected, as antigenbearing DCs will migrate to lymph nodes, where lymph node-residing DCs may influence any immunological effect $(14,30)$. This behavior, chemotaxis and chemoattraction of other DCs and the complex interplay between different DC classes, may explain the absence of differences in immune response as function of the injection depth. Contrarily to rat skin though, in human skin the epidermis has a thickness of 50-100 $\mu \mathrm{m}(30)$. As the redesigned applicator is able to inject at a depth of $50 \mu \mathrm{m}$, injection directly into the human epidermis is possible. This allows to study LC function in intradermal immunity in the future. In conclusion, our applicator could provide a method to investigate depth-dependent intradermal vaccine immunogenicity in human.

Furthermore, results presented in this study are important in microneedle patch design for intradermal IPV immunization as the independence of IPV1 immunization on skin depth suggests that microneedle length will not affect the immune response and thus precise dosing at a certain skin depth is not 
required. This may put a smaller burden on the design specifications of microneedle applicators.

Besides, an effort was made to find a dose sparing strategy for IPV immunization. However, intradermal and intramuscular immunization without use of adjuvants led to similar results, likewise as reported for other microneedle strategies: HMN in rats $(8,11)$ or humans (13), coated microneedles in rats (9), dissolving microneedles in rats (10) or rhesus macaques (12). Furthermore, clinical trials in humans have been performed to investigate IPV dose sparing, by comparison of a $80 \%$ reduced IPV dose (20\% of full dose) administered intradermally against a full IPV dose administered intramuscularly. For polio prime immunization in newborn infants, intradermal 20\% IPV dose resulted in similar $(4,5)$ or inferior (31-33) seroconversion rates compared to a full intramuscular dose. Similarly, for polio booster immunization, intradermal $20 \%$ IPV dose resulted in either similar $(6,13)$ or inferior $(34,35)$ seroconversion rates in comparison to full intramuscular dose. However, intradermal immunization generally resulted in significantly lowered antibody titers (5,6,31-35). These findings were seemingly not dependent on the intradermal immunization method used, because jet injectors $(5,6,31,32,34,35)$, the Mantoux technique $(4,35)$ or HMN arrays $(13,33)$ evenly resulted in either similar or inferior results to full intramuscular dose. In this study, it is shown that the depth at which the antigen was administered by these different intradermal immunization techniques cannot explain this phenomenon either. However, the $80 \%$ dose-reduction goal may be unrealistic, as a $60 \%$ dose-reduction was achieved by HMN mediated intradermal immunization without use of adjuvants $(11,13)$.

Adjuvants may be used as another strategy for IPV dose sparing (3). For example, aluminum hydroxide $(16,17)$ and aluminum phosphate $(18,19)$ have been successfully used as an adjuvant for intramuscular IPV immunization. Because aluminum salts cannot be administered intradermally due to local adverse effects, adjuvants $\mathrm{CpG}$ and $\mathrm{CT}$ were assessed for intradermal IPV1 immunization. Both CpG and CT have shown to be safe as adjuvant in intradermal immunization in humans $(36,37)$. In the present study, immuneenhancing effects on IPV1-specific IgG responses by the adjuvants $\mathrm{CpG}$ and $\mathrm{CT}$ were significant, which indicates that $\mathrm{CpG}$ and $\mathrm{CT}$ might be potential adjuvant candidates for intradermal IPV1 immunization and may lead to dose sparing. Indeed, CpG was reported by others to be an effective immune enhancing adjuvant for IPV immunization (38). Although this latter study utilized mice and a different $\mathrm{CpG}$ motif (ODN2006), both studies report on the potential for CpG as adjuvant in IPV immunization. Furthermore, we have reported a 6.9- and 7.3-fold increase in IPV1specific IgG serum titers for intradermally IPV co- administered with CpG and CT, respectively. This increase was larger as reported for intradermal IPV immunization in mice in combination with CpGODN2006 (4-fold) or double mutant heat-labile enterotoxin from E. coli LT (R192G/L211A) (2.5-fold) $(38,39)$. Contrarily, a 10 -fold increase was achieved by CAF01 liposomal IPV formulation for intradermal IPV immunization in mice (40).

\section{CONCLUSION}

In this study, an unique dose sparing strategy for IPV 1 was investigated by intradermal microinjections of small volumes of IPV1 formulation at different predetermined depths in rat skin in vivo. To enable this, a HMN applicator controllable in injection rate, -volume and -depth was developed that allowed for intradermal microinjections. Results indicated however, that intradermal immunogenicity was not dependent on the injection depth. Nonetheless, IPV1 immunization by minimally-invasive intradermal microinjections resulted in similar IPV1-specific antibody responses in comparison to IPV1 immunization by more invasive and painful intramuscular injections. Moreover, intradermal IPV1 immunization antibody responses were significantly increased (7-fold) by adjuvants CpG and CT, such that a single minimally-invasive intradermal immunization by HMN resulted in comparable antibody responses to two intramuscular immunizations.

\section{ACKNOWLEDGMENTS AND DISCLOSURES}

The authors thank Heleen Kraan for contributions to analysis of IPV-specific IgG and VN responses, Raphael Zwier for contributions to the applicator design and Anne Römgens for contributions to discussions related to this study.

\section{COMPLIANCE WITH ETHICAL STANDARDS}

Funding This study was funded by the Dutch Technology Foundation STW, which is part of the Netherlands Organization for Scientific Research (NWO), and which is partly funded by the Ministry of Economic Affairs (project no. 11259).

Conflict of Interest The authors declare that they have no conflict of interest.

Ethical Approval All applicable international, national, and/or institutional guidelines for the care and use of animals were followed. This article does not contain any studies with human participants performed by any of the authors. 


\section{REFERENCES}

1. Kew OM, Sutter RW, de Gourville EM, Dowdle WR, Pallansch MA. Vaccine-derived polioviruses and the endgame strategy for global polio eradication. Annu Rev Microbiol. 2005;59:587-635.

2. World Health Organization W. Polio eradication and endgame strategic plan 2013-2018. Geneva: World Health Organization (WHO); 2013.

3. Okayasu H, Sutter RW, Jafari HS, Takane M, Aylward RB. Affordable inactivated poliovirus vaccine: strategies and progress. J Infect Dis. 2014;210 Suppl 1:S459-64.

4. Cadorna-Carlos J, Vidor E, Bonnet MC. Randomized controlled study of fractional doses of inactivated poliovirus vaccine administered intradermally with a needle in the Philippines. Int J Infect Dis. 2012;16(2):e1 10-6.

5. Mohammed AJ, AlAwaidy S, Bawikar S, Kurup PJ, Elamir E, Shaban MM, et al. Fractional doses of inactivated poliovirus vaccine in Oman. N Engl J Med. 2010;362(25):235 1-9.

6. Soonawala D, Verdijk P, Wijmenga-Monsuur AJ, Boog CJ, Koedam P, Visser LG, et al. Intradermal fractional booster dose of inactivated poliomyelitis vaccine with a jet injector in healthy adults. Vaccine. 2013;31(36):3688-94.

7. van der Maaden K, Jiskoot W, Bouwstra J. Microneedle technologies for (trans)dermal drug and vaccine delivery. J Control Release. 2012;161(2):645-55

8. van der Maaden K, Trietsch SJ, Kraan H, Varypataki EM, Romeijn S, Zwier R, et al. Novel hollow microneedle technology for depth-controlled microinjection-mediated dermal vaccination: a study with polio vaccine in rats. Pharm Res. 2014;31(7):1846-54.

9. van der Maaden K, Sekerdag E, Schipper P, Kersten G, Jiskoot W, Bouwstra JA. Layer-by-layer assembly of inactivated poliovirus and $\mathrm{N}$-trimethyl chitosan on $\mathrm{pH}$-sensitive microneedles for dermal vaccination. Langmuir. 2015.

10. Kraan H, Ploemen I, van de Wijdeven G, Que I, Lowik C, Kersten $\mathrm{G}$, et al. Alternative delivery of a thermostable inactivated polio vaccine. Vaccine. 2015;33(17):2030-7.

11. Kouiavskaia D, Mirochnitchenko O, Dragunsky E, Kochba E, Levin Y, Troy S, et al. Intradermal inactivated poliovirus vaccine: a preclinical dose-finding study. J Infect Dis. 2015;211(9):1447-50.

12. Edens G, Dybdahl-Sissoko NC, Weldon WC, Oberste MS, Prausnitz MR. Inactivated polio vaccination using a microneedle patch is immunogenic in the rhesus macaque. Vaccine. 2015.

13. Troy SB, Kouiavskaia D, Siik J, Kochba E, Beydoun H, Mirochnitchenko $\mathrm{O}$, et al. Comparison of the immunogenicity of various booster doses of inactivated polio vaccine delivered intradermally versus intramuscularly to HIV-infected adults. J Infect Dis. 2015;211(12):1969-76.

14. Combadiere B, Liard C. Transcutaneous and intradermal vaccination. Hum Vaccin. 201 1;7(8):811-27.

15. Hawken J, Troy SB. Adjuvants and inactivated polio vaccine: a systematic review. Vaccine. 2012;30(49):6971-9.

16. Auerswald W, Juergenssen O. On the problem of antibody formation against poliomyelitis in small children. Studies on the effect of an adjuvant vaccine with antigen components against poliomyelitis, diphtheria, pertussis and tetanus. Wien Med Wochenschr. 1960;110:646-8

17. Li RG, Li FX, Li YP, Hou QM, Li CG, Li YN, et al. Immunogenicity and safety of a pentavalent acellular pertussis combined vaccine including diphtheria, tetanus, inactivated poliovirus and conjugated Haemophilus Influenzae type b polysaccharide for primary vaccination at 2, 3, 4 or 3, 4, 5 months of age in infants in China. Vaccine. 2011;29(10):1913-20.
18. Yeh SH, Ward JI, Partridge S, Marcy SM, Lee H, Jing J, et al. Safety and immunogenicity of a pentavalent diphtheria, tetanus, pertussis, hepatitis $\mathrm{B}$ and polio combination vaccine in infants. Pediatr Infect Dis J. 2001;20(10):973-80.

19. Guerra FA, Blatter MM, Greenberg DP, Pichichero M, Noriega FR, Study G. Pentacel. Safety and immunogenicity of a pentavalent vaccine compared with separate administration of licensed equivalent vaccines in US infants and toddlers and persistence of antibodies before a preschool booster dose: a randomized, clinical trial. Pediatrics. 2009;123(1):301-12.

20. Gupta RK. Aluminum compounds as vaccine adjuvants. Adv Drug Deliv Rev. 1998;32(3):155-72.

21. Albrecht P, van Steenis G, van Wezel AL, Salk J. Standardization of poliovirus neutralizing antibody tests. Rev Infect Dis. 1984;6 Suppl 2:S540-4.

22. Westdijk J, Koedam P, Barro M, Steil BP, Collin N, Vedvick TS, $e t$ al. Antigen sparing with adjuvanted inactivated polio vaccine based on Sabin strains. Vaccine. 2013;31(9):1298-304.

23. Martanto W, Moore JS, Kashlan O, Kamath R, Wang PM, O’Neal JM, et al. Microinfusion using hollow microneedles. Pharm Res. 2006;23(1):104-13.

24. Wang PM, Cornwell M, Hill J, Prausnitz MR. Precise microinjection into skin using hollow microneedles. J Invest Dermatol. 2006;126(5):1080-7.

25. Roxhed N, Samel B, Nordquist L, Griss P, Stemme G. Painless drug delivery through microneedle-based transdermal patches featuring active infusion. IEEE Trans Biomed Eng. 2008;55(3):1063-71.

26. Hafeli UO, Mokhtari A, Liepmann D, Stoeber B. In vivo evaluation of a microneedle-based miniature syringe for intradermal drug delivery. Biomed Microdevices. 2009;1 1(5):943-50.

27. Burton SA, Ng CY, Simmers R, Moeckly C, Brandwein D, Gilbert $\mathrm{T}$, et al. Rapid intradermal delivery of liquid formulations using a hollow microstructured array. Pharm Res. 2011;28(1):31-40.

28. Kim YC, Park JH, Prausnitz MR. Microneedles for drug and vaccine delivery. Adv Drug Deliv Rev. 2012;64(14):1547-68.

29. Roxhed N, Griss P, Stemme G. Membrane-sealed hollow microneedles and related administration schemes for transdermal drug delivery. Biomed Microdevices. 2008;10(2):271-9.

30. Hirschberg HJ, van Riet E, Oosterhoff D, Bouwstra JA, Kersten GF. Animal models for cutaneous vaccine delivery. Eur J Pharm Sci. 2015;71:112-22.

31. Resik S, Tejeda A, Lago PM, Diaz M, Carmenates A, Sarmiento L, et al. Randomized controlled clinical trial of fractional doses of inactivated poliovirus vaccine administered intradermally by needle-free device in Cuba. J Infect Dis. 2010;201(9):1344-52.

32. Resik S, Tejeda A, Sutter RW, Diaz M, Sarmiento L, Alemani N, et al. Priming after a fractional dose of inactivated poliovirus vaccine. N Engl J Med. 2013;368(5):416-24.

33. Anand A, Zaman K, Estivariz CF, Yunus M, Gary HE, Weldon WC, et al. Early priming with inactivated poliovirus vaccine (IPV) and intradermal fractional dose IPV administered by a microneedle device: a randomized controlled trial. Vaccine. 2015;33(48): 6816-22.

34. Estivariz CF, Jafari H, Sutter RW, John TJ, Jain V, Agarwal A, et al. Immunogenicity of supplemental doses of poliovirus vaccine for children aged 6-9 months in Moradabad, India: a communitybased, randomised controlled trial. Lancet Infect Dis. 2012;12(2): 128-35.

35. Resik S, Tejeda A, Mach O, Fonseca M, Diaz M, Alemany N, et al. Immune responses after fractional doses of inactivated poliovirus vaccine using newly developed intradermal jet injectors: a randomized controlled trial in Cuba. Vaccine. 2015;33(2):307-13.

36. Scheiermann J, Klinman DM. Clinical evaluation of CpG oligonucleotides as adjuvants for vaccines targeting infectious diseases and cancer. Vaccine. 2014;32(48):6377-89. 
37. Eypper EH, Johnson PV, Purro EI, Hohmann EL. Transcutaneous immunization of healthy volunteers with an attenuated Listeria monocytogenes vaccine strain and cholera toxin adjuvant. Vaccine. 2013;31(32):3257-61.

38. Yang G, Shi H, Zhou J, Liang Y, Xu H. CpG oligodeoxynucleotides are a potent adjuvant for an inactivated polio vaccine produced from Sabin strains of poliovirus. Vaccine. 2009;27(47):6558-63.
39. Norton EB, Bauer DL, Weldon WC, Oberste MS, Lawson LB, Clements JD. The novel adjuvant dmLT promotes dose sparing, mucosal immunity and longevity of antibody responses to the inactivated polio vaccine in a murine model. Vaccine. 2015;33(16):1909-15.

40. Dietrich J, Andreasen LV, Andersen P, Agger EM. Inducing dose sparing with inactivated polio virus formulated in adjuvant CAF01. PLoS One. 2014;9(6), e100879. 